Daily COVID-19 Cases and Test Positivity Rates Against Vaccination Rates in US States, Puerto Rico, and D.C. (08-08-2021)

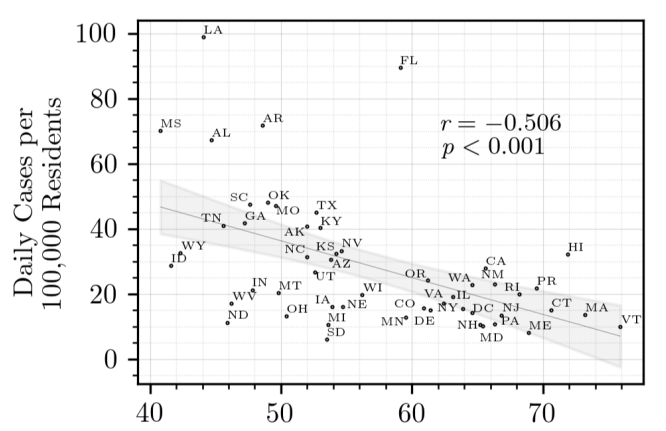

Percentage of Population Vaccinated $(\geq 1$ Dose $)$ 100

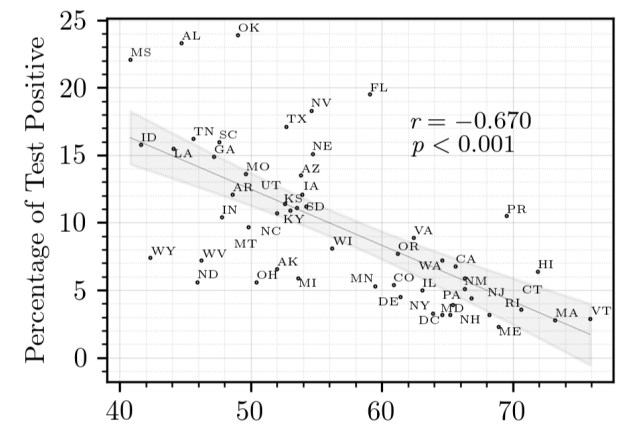

Percentage of Population Vaccinated ( $\geq 1$ Dose) $2 5 \longdiv { \mathrm { AL } }$

\title{
Correlations Between COVID-19 Cases, Test Positivity Rates, and Vaccinations in the United States
}

Floe Foxon ${ }^{1}$

1 Pinney Associates

Funding: The author(s) received no specific funding for this work.

Potential competing interests: The author(s) declared that no potential competing interests exist.

\section{Abstract}

The present study reports methods used to determine simple linear geographic correlations between COVID-19 vaccination rates, and both daily per capita COVID-19 cases and COVID test positivity rates in US states as well as D.C. and Puerto Rico. Cases and positivity rates were negatively associated with vaccination rates, which is unsurprising and consistent with longitudinal studies which have demonstrated significant COVID vaccine efficacy against transmission, though it remains to be seen how the emergence of more transmissible variants will affect these associations. Associations did not differ significantly for one or more vaccine doses versus two doses only.

\section{Methods}

Data

All data were sourced from the Act Now Coalition's U.S. COVID Risk \& Vaccine Tracker, ${ }^{[1]}$ which combines data from the CDC COVID Data Tracker, ${ }^{[2]}$ the Department of Health and Human Services COVID-19 PCR Testing Time Series dataset, ${ }^{[3]}$ and the New York Times COVID-19 Data Repository. ${ }^{[4]}$ In each state/territory/district, daily new cases were defined as the number of new COVID cases per day, averaged 
across the last seven days, divided by population of the locality and multiplied by 100,000 . Positive test rates in each locality were defined as the number of positive PCR tests across the last seven days, divided by the total number of PCR tests across the last seven days. Vaccination rates were simply the percent of the total population (all ages) in each locality who had received a COVID-19 vaccine authorized for emergency use by the US Food and Drug Administration, which included the BNT162b2 (Pfizer-BioNTech), mRNA-1273 (Moderna), and Ad26.COV2.S (Janssen) vaccines. Additional data details are provided on the Act Now Coalition's methodology page. ${ }^{[5]}$ Data were collected on August 8, 2021 and are available in the Supplementary Data.

Analyses

For each locality, daily cases and positive test rates were plotted against the percentage of the population vaccinated with one or more doses and with two doses. Pearson correlation coefficients were calculated with a significance level of $\alpha=0.05$. For illustrative purposes, simple linear regressions were modeled as $y=b_{0}+b_{1} x$ with $90 \%$ confidence intervals. The null hypothesis that the regression gradients were the same for one or more doses and for two doses, that is $H_{0}: b_{1}=b_{2}$, was tested with the normal $z$ test

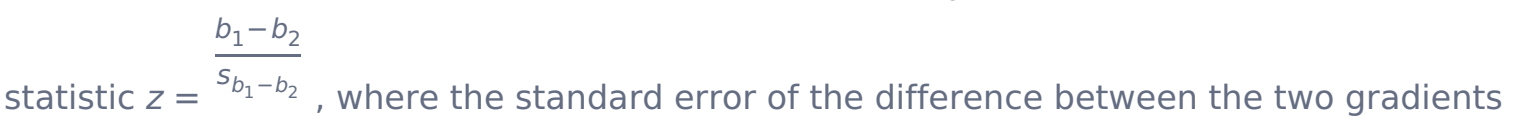

$s_{b_{1}-b_{2}}=\sqrt{s_{b_{1}}^{2}+s_{b_{2}}^{2}}$, and the $p$-value is two-tailed. All analyses were conducted in Python version 3.7.6 with the packages Numpy version 1.18.5, Pandas version 1.0.5, Matplotlib version 3.2.2, Scipy version 1.5.0, Uncertainties version 3.1.2, and adjustText version 0.7.3.

\section{Results \& Discussion}

Figure 1 shows the correlations between vaccination rates and both cases and test positivity. Both cases and positivity rates were significantly and negatively associated with vaccination rates across the US. The z-test results failed to reject the null hypothesis that the regression gradients were equal for one or more doses and for two doses.

Figure 1: Daily COVID-19 Cases and Test Positivity Rates Against Vaccination Rates in US States, Puerto Rico, and D.C. (08-08-2021) 


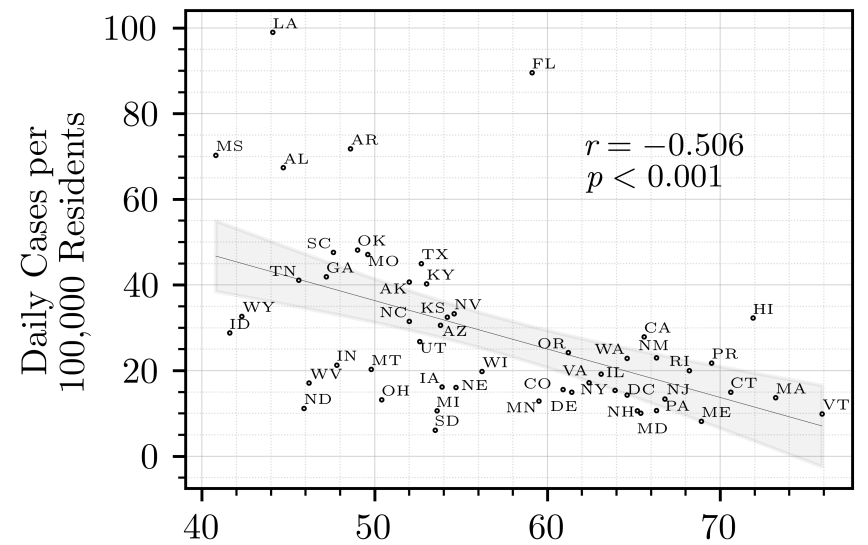

Percentage of Population Vaccinated ( $\geq 1$ Dose)

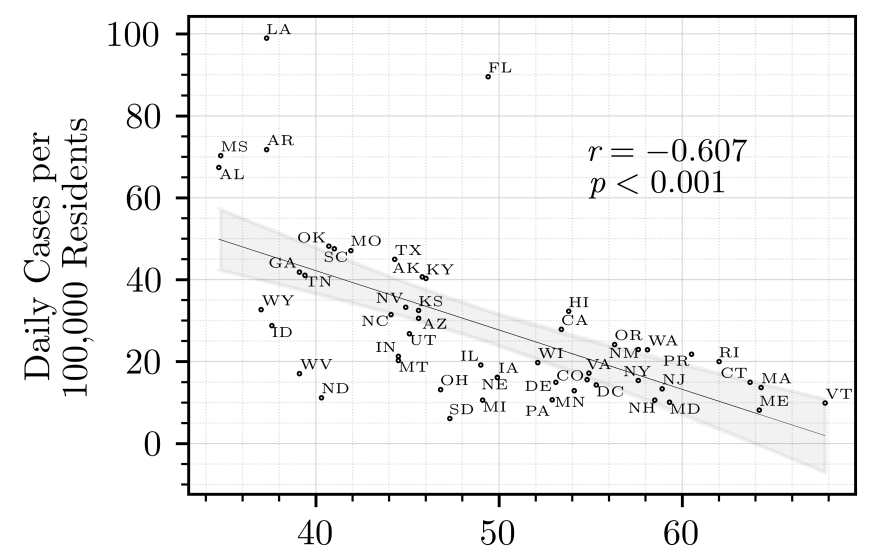

Percentage of Population Vaccinated (2 Doses)

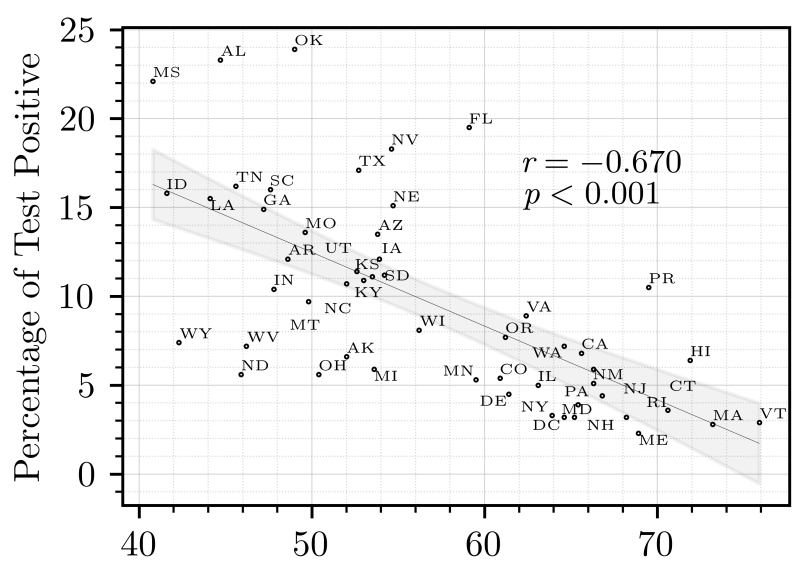

Percentage of Population Vaccinated ( $\geq 1$ Dose)

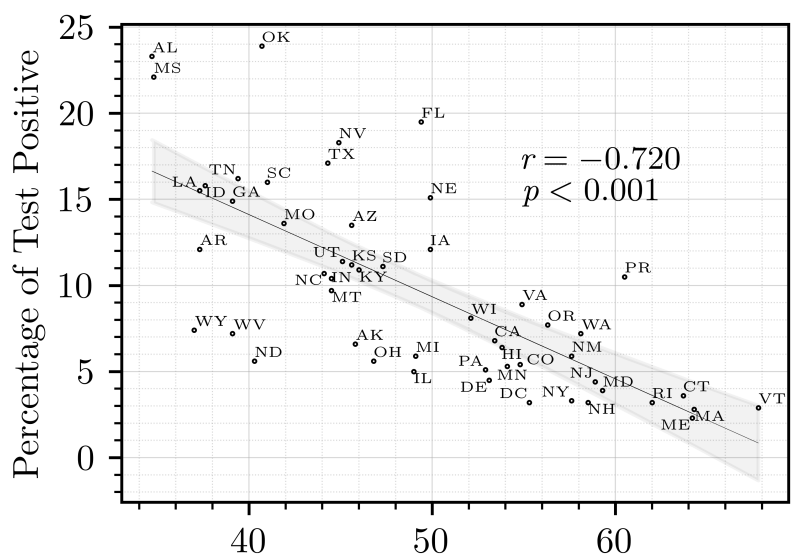

Percentage of Population Vaccinated (2 Doses)

Though not proof of causation, these results may be consistent with extant literature demonstrating that current COVID-19 vaccines are efficacious in reducing SARS-CoV-2 transmission. One study applying RCT data for the mRNA-1273 vaccine to simulated Bernoulli trials found that vaccinated individuals had $\leq 61 \%$ reduced probability of transmitting infection, using a vaccinated person's reduced probability of harboring SARS-CoV-2 at a given time (the vaccine's efficacy for viral positivity) as an upper bound for the vaccine's efficacy against transmission. ${ }^{[6]}$ Similar results were obtained in a Public Health England (PHE) study using England's Household Transmission Evaluation Dataset (HOSTED). Compared to unvaccinated individuals, vaccinated individuals who became infected three weeks after receiving the BNT162b2 or ChAdOx1 nCoV19 (Oxford-AstraZeneca) vaccines were 38 - 49\% less likely to transmit SARS-CoV-2 to household contacts. ${ }^{[7]}$ Most recently, a study with data from the Netherlands estimated that fully vaccinated individuals were $71 \%$ less likely to transmit the virus to household contacts (58\% for ChAdOx1 nCoV-19, 70\% for BNT162b2, 77\% for Ad26.COV2.S, and 88\% for mRNA-1273). ${ }^{[8]}$ The mechanism by which these vaccines are thought to reduce transmission is decreased viral load in vaccinated infected individuals, ${ }^{[9]}$ since viral load has been identified as a leading driver of SARS-CoV-2 transmission. ${ }^{[10]}$ Notably however, these studies contain primarily data from the first half of 2021, and so do not account for changes in transmission of SARS-CoV-2 due to the appearance of new variants. On August 6, 2021, both 
PHE scientists and CDC Director Dr. Rochelle Walensky warned of decreased vaccine efficacy against transmission due to increased transmissibility of the B.1.617.2 (Delta) SARS-CoV-2 variant, ${ }^{[11][12]}$ which has become dominant in the UK ${ }^{[13]}$ and US. ${ }^{[14]}$ A more recent study focusing on the B.1.617.2 variant has suggested similar viral loads in both vaccinated and unvaccinated infected individuals, ${ }^{[15]}$ which could reduce current vaccine efficacy against transmission. It has also been demonstrated experimentally since before the COVID-19 pandemic that vaccines with high efficacy against death but low efficacy against transmission could create conditions within individuals which enhance the emergence of hyperpathogenic virus strains, ${ }^{[16]}$ and this has been cited most notably by influential virologist Geert Vanden Bossche in an open letter to the World Health Organization. ${ }^{[17]}$ This effect has not been demonstrated for SARS-CoV-2 in humans, though preliminary research has suggested that vaccine-driven SARS-CoV-2 virulence evolution is plausible but not definite, and that in this event COVID vaccines would achieve herd immunity regardless if administered to a large enough proportion of the population. ${ }^{[18]}$ The present study may suggest that so far, vaccine roll-outs have been successful in reducing virus transmission as measured by cases and positive test rates. However, it is crucial that these vaccines continue to be administered to a significant percentage of the population in a timely manner.

\section{Limitations}

Cases and test positivity rates may be influenced by local testing capacity and surge testing in communities with high prevalence of SARS-CoV-2. Data are assumed to be temporally consistent though reporting lags may influence findings. Simple correlations and simple regression models do not account for confounding by differences in lockdowns, mask mandates, and other coronavirus mitigation strategies between locations. Findings may not be extrapolated into the future due to changes in transmissibility from new variants. Future studies should seek to replicate these findings while controlling for confounders and stratify models by variant.

\section{References}

1. ^Act Now Coalition. , , , , et al. (2021). U.S. COVID Risk \& Vaccine Tracker.

2. 'CDC. , , . (2021). COVID Data Tracker.

3. ^HHS. , , . (2021). COVID-19 Diagnostic Laboratory Testing (PCR Testing) Time Series.

4. ^NYT. , , . (2021). COVID-19 Data Repository.

5. ^Act Now Coalition. , , , , et al. (2021). Risk levels \& key metrics.

6. ^Marc Lipsitch, Rebecca Kahn. (2021). Interpreting vaccine efficacy trial results for infection and transmission. Vaccine, vol. 39 (30), 4082-4088. doi:10.1016/j.vaccine.2021.06.011.

7. ^Ross J. Harris, Jennifer A. Hall, Asad Zaidi, Nick J. Andrews, et al. (2021). Effect of Vaccination on Household Transmission of SARS-CoV-2 in England. N EnglJ Med. doi:10.1056/nejmc2107717.

8. `Brechje de Gier, Stijn Andeweg, Rosa Joosten, Ronald ter Schegget, et al. (2021). Vaccine effectiveness against SARS-COV-2 transmission and infections among household and other close contacts of 
confirmed cases, the Netherlands, February to May 2021. doi:10.2807/1560-

7917.es.2021.26.31.2100640.

9. `Matan Levine-Tiefenbrun, Idan Yelin, Rachel Katz, Esma Herzel, et al. (2021). Initial report of decreased SARS-CoV-2 viral load after inoculation with the BNT162b2 vaccine. Nat Med, vol. 27 (5), 790-792. doi:10.1038/s41591-021-01316-7.

10. 'Michael Marks, Pere Millat-Martinez, Dan Ouchi, Chrissy h Roberts, et al. (2021). Transmission of COVID-19 in 282 clusters in Catalonia, Spain: a cohort study. The Lancet Infectious Diseases, vol. 21 (5), 629-636. doi:10.1016/s1473-3099(20)30985-3.

11. `CNN. , , . (2021). Fully vaccinated people who get a Covid-19 breakthrough infection can transmit the virus, CDC chief says.

12. ^Reuters. , , , , et al. (2021). Early signs COVID-19 vaccines may not stop Delta transmission, England says.

13. ^PHE. , , . (2021). Variants: distribution of case data, 6 August 2021.

14. ${ }^{\wedge} C D C$. , , . (2021). CDC COVID Data Tracker Variant Proportions 4/25/2021 - 7/31/2021.

15. `Kasen K. Riemersma, Brittany E. Grogan, Amanda Kita-Yarbro, Gunnar E. Jeppson, et al. (2021). Vaccinated and unvaccinated individuals have similar viral loads in communities with a high prevalence of the SARS-CoV-2 delta variant. doi:10.1101/2021.07.31.21261387.

16. AAndrew F. Read, Susan J. Baigent, Claire Powers, Lydia B. Kgosana, et al. (2015). Imperfect Vaccination Can Enhance the Transmission of Highly Virulent Pathogens. PLoS Biol, vol. 13 (7), e1002198. doi:10.1371/journal.pbio.1002198.

17. `Geert Vanden Bossche. , , , , et al. (2021). Open Letter to WHO.

18. 'Ian F. Miller, C. Jessica E. Metcalf. (2020). Assessing the risk of vaccine-driven virulence evolution in SARS-CoV-2. doi:10.1101/2020.12.01.20241836. 